\title{
El gasto militar: factor estratégico para la modernización de las fuerzas armadas
}

\author{
TCCIM Diego Fernando Patrón Cáceres ${ }^{1}$
}

\begin{abstract}
Resumen
El presente estudio es una revisión documental de las principales estrategias vinculadas con el gasto militar en Colombia. Para la consecución del objetivo planteado se recuren a fuentes secundarias. El artículo consta de dos fases una primera donde se realiza un acercamiento a la importancia de la innovación y la tecnología en el ámbito militar, como garante de la modernización de las fuerzas militares y la segunda fase se relaciona con el gasto militar como factor clave de la modernización. Dentro de las principales conclusiones se puede decir que el estado colombiano de velar por el establecimiento de estrategias direccionada a la inversión en la modernización de las fuerzas militares, desde las aristas tecnológicas, armamentista, educativa entre otras; con el fin de logra una fuerza armada con capacidades afrontar retos presentes y futuros en materia de defensa, seguridad y soberanía.
\end{abstract}

\section{Palabras Claves}

Gasto militar, seguridad pública, defensa, Innovación tecnológica, economía de defensa.

\begin{abstract}
This study is a documentary review of the main strategies related to military spending in Colombia. In order to achieve the stated objective, recourse to secondary sources. The article consists of two phases, a first one where an approach is made to the importance of innovation and technology in the military field, as guarantor of the modernization of military forces and the second phase is related to military spending as a key factor in Modernization Among the main conclusions it can be said that the Colombian state of ensuring the establishment of strategies aimed at investment in the modernization of military forces, from the technological, arms, educational, and other aspects; in order to achieve an armed force with capabilities to face present and future challenges in defense, security and sovereignty.
\end{abstract}

\section{Keywords}

Military spending, public safety, defense, Technological innovation, defense economy

1. Teniente coronel de la infantería de marina 


\section{Introducción}

Las estrategias son medios de consecución de objetivos, garantizando a través de ella el éxito organizacional (De Ríos, 2018); las estrategias en el sector militar se convierten en el medio efectivo para afrontar los conflictos. Una de las estrategias de mayor incidencia en el éxito de las tropas es la vinculada con la innovación tecnológica, la cual tiene su origen en hacer frente a los desafíos impuestos por la globalización de la economía, por los avances tecnológicos y la expansión del uso de nuevas técnicas de manejo de información y comunicaciones (Hernández, Cardona, \& Del Rio, 2017).

En este contexto es necesaria la actualización de la estrategia de innovación tecnológica implementada en el ambiro de seguridad y defensa, con el único fin de contar con una fuerza armada de vanguardia, con capacidad de velar por la soberanía tanto en tiempo de guerra como paz. Un efecto subsecuente de la innovación tecnología de las fuerzas armadas es la reducción de la incertidumbre mediante el aumento de las capacidades de respuesta no solo en terrenos ya conocidos sino también en el ultraterrestre y el ciberespacio (Perez Arrieu, 2017).

La demanda de capacidades de innovación y tecnología en futuros escenarios de conflicto necesitará mayor flexibilidad y capacidad de incorporación con el fin de poder hacer frente a amenazas imprevistas (Mayo, 2011).

Como parte de sus responsabilidades el estado de propender por el fortalecimiento de las fuerzas armadas, para el cumplimiento de su objetivo relacionado con el mantenimiento de una nación libre de amenazas; por ello el poderío militar de una nación requiere de una inyección presupuestal constante que lo fortalezca, modernice, la tecnifique y la dote de tecnología de punta.

Un factor que juega un gran papel en la modernización de las fuerzas militares es la prospectiva, pues a partir de esta se prevén los posibles escenarios y en consecuencia con ello se desarrolla la planeación presupuestal y defensa de la nación.

\section{Metodología}

El enfoque de la investigación es cuantitativo y el alcance es descriptivo; se realizó la recopilación de los documentos vinculados con innovación y gasto militar, a partir de dicha fuente secundaria se concluyeron cuáles son las principales estrategias del gasto militar implementadas para la modernización de las fuerzas militares.

\section{Resultados}

\section{Antecedentes Gasto Público.}

El estado ha afrontado muchas crisis económicas, las cuales han afectado el presupuesto de gastos de la nación y con ello la ejecución de los planes de desarrollo, para lo cual el gobierno se vio abocado a reajustar el presupuesto de gasto público e implementar reformas tributarias para recuperar ingresos que el estado dejo de percibir. Más allá de la efectividad de las medidas de carácter tributario es necesaria la sinergia de todos los elementos del estado con la finalidad de ser más efectivos en la generación de ingresos y la efectiva administración del gasto, todo ello fundamentado en la sostenibilidad fiscal del país.

Algunos casos particulares han demostrado el intento del estado por maniobrar con situaciones de crisis en el gasto más exactamente el defensa; entre el año de 1994 a 1998 en el periodo presidencial de Ernesto Samper, e emitieron bonos de seguridad, los cuales ayudaron a garantizar la financiación de las fuerzas militares. En el periodo comprendido entre 2002 a 2010 bajo el mandato de Álvaro Uribe Vélez, se implementaron diversos mecanismo para garantizar la política de seguridad democrática, entre ellos se pueden mencionar: la aplicación de impuestos al patrimonio tanto de personas naturales como jurídicas; posteriormente se implementa un impuesto especial de financiamiento de la seguridad democrática, a través del cual se garantizaba la ejecución de estrategias en materia de innovación y desarrollo tecnológico.

\section{Actualización tecnológica}

A diferencia de la tecnología utilizada por los civiles la tecnología militar conlleva la aplicación 
de tecnologías basadas en las rigurosas especificaciones o requerimientos demandados por las tropas para hacer frente a situaciones de conflicto; ello implica muchas veces la restructuración de tecnología civil bajo los parámetros más estrictos y exigentes. Una forma de lograr avances en materia de tecnología es a través de tratados cooperación internacionales con países potencias.

La actualización no percibirse como un costo adicional en períodos futuros, excepto cuando se requiere extender la vida útil del material estratégico como fueron los casos de vehículos blindados del Ejército Nacional (2002-2005), fragatas misileras y submarinos oceánicos (2011-2014), en tal sentido se proyectan como costos de ciclo de vida generados por decisiones de gestión de los recursos, costos asociados al sostenimiento de equipos adquiridos y con extensión de vida útil.

\section{Gasto Militar}

EL gasto militar es comprendido de diversas maneras, cada nación lo ve de acuerdo a sus instituciones y ordenamientos jurídicos propios, pero en general, es el gasto público destinado por una nación a sus Fuerzas Militares para su protección frente a las amenazas externas. En Colombia, esta definición se complementa con la responsabilidad que reciben las Fuerzas Armadas dentro de la situación de conflicto interno. (Gutiérrez, 1995)

Esto nos indica que el Gasto en Defensa y Seguridad o Gasto Militar, no solo incluye la necesidad de la defensa del país contra amenazas extrajeras, sino que también incluye el gasto en seguridad contra amenazas domésticas.

\section{Reducción del gasto militar y Estrategias del sector defensa}

En primera instancia hay que comprender que el concepto de estrategia es un término que se encuentra en todas y cada uno de las áreas de las organizaciones, por ejemplo: para (Sarmiento, 2018), las estrategias son herramientas que permiten fortaleza, desarrollo de competitividad y productividad de las empresas. Por su parte (Rubio, 2018), asegura que las estrategias permiten afrontar las situaciones cambiantes en relación con la consecución de las operaciones; desde otra perspectiva (Peinado, 2018) afirma que la estrategia establece la base para la formulación de planes y proyectos a mediano y largo plazo; así mismo para (Vergara, et al, 2019), las estrategias deben entrañar en si misma un carácter evolutivo e innovador que permita a las organizaciones estar a la vanguardia.

Para Chandler (1962), la estrategia es componente que establece los fines elementales de una organización en el largo plazo, de igual manera, la elección de un paquete de medidas de acciones y la distribución de los recursos necesarios para alcanzar dichos objetivos. Por su parte, Ansoff (1965) concibió la estrategia como un conjunto de acciones lógicas entre la naturaleza de la empresa y los productos o bienes y servicios ofrecidos en su mercado, es decir, que la estrategia permite articular las actividades productivas de la empresa acorde con los requerimientos del mercado.

Miles \& Snow (1978) definieron la estrategia como el marco de decisiones y acciones que se requiere adoptar para facilitar el alineamiento progresivo de una organización con el entorno y Porter (1980) sugería que las estrategias están definidas por como adquirir o perpetuar una ventaja competitiva dentro del mercado.

\section{Conclusiones}

En la actualidad es muy común que las organizaciones se desarrollen tecnológicamente compartiendo su conocimiento a través de alianzas, constituyendo relaciones contractuales, formales o informales. Esto permitirá comenzar nuevos ciclos de aprendizaje y de conocimiento en todos los procesos, donde podrá hablarse de un "sistema de Conocimiento e Innovación de la Defensa Nacional”, con énfasis en aspectos tecnológicos, sociales y económicos.

Estos conceptos están considerados dentro de la Economía de la defensa, de forma asociada con la economía de la empresa que tiene relación con defensa o industria de defensa. Estas relaciones se enmarcan en la cooperación entre organizaciones, alianzas estratégicas, gestión del conocimiento, innovación y tecnología dual. 
De igual forma, el Estado mantiene a las Fuerzas Militares a la vanguardia mundial, sosteniendo la inversión con el fin de mantenerlas dotas y preparadas, garantizando la seguridad y defensa de la población, el territorio y sus fronteras, y manteniendo la innovación, actualización e innovación tecnológica, que les permita atender nuevos fenómenos delincuenciales.

Adicionalmente, es posible concluir que el cambio en la estrategia de seguridad y defensa del Estado es necesario para proyectar profundos cambios en la forma como el Estado puede garantizar los recursos necesario para mantener y sostener sus Fuerzas Armadas para estar preparadas en los nuevos escenarios, reduciendo los costos, y generando valor socio económico.

Analizar de manera permanente los factores de producción del sector Defensa que le permitan competir en mercados nacionales e internacionales, creando y produciendo bienes y servicios, como parte de su oferta.

Definir la permanencia o no de la nación en sectores internacionalmente competitivos, relacionados con la Defensa y cooperación para el Desarrollo regional y mundial, al respecto, aportar a organismos como la Organización de Naciones Unidas (ONU) y Organización del Tratado del Atlántico Norte (OTAN), participando en operaciones de paz, que pueden retribuirse con experiencia, modernización y actualización del sector.

Establecer para el sector Defensa, condiciones del Estado para que las empresas del Grupo Social y Empresarial de la Defensa (GSED) se creen, organicen y gestionen, estableciendo competencia interna y externa con otros sectores de la economía.

Establecer políticas que presionen sobre la estructura organizacional del sector Defensa la innovación tecnológica permanente en todos sus procesos, lo cual les daría ventaja competitiva que sería mejorada a lo largo del tiempo. Algunos principios básicos para desempeñar un papel de apoyo a la competitividad son alentar al cambio, promover la rivalidad interior, estimular la innovación.
El Estado tiene el papel más importante en el direccionamiento de políticas económicas y de relaciones internacionales para el sostenimiento y optimización del gasto Militar, sus decisiones son esenciales para ayudar o apoyar la industria, estableciendo lineamientos para contribuir con la competitividad de los sectores estratégicos u objetivos fijados. El entorno que generan sus decisiones estimula el logro de ventajas competitivas. Tiene un papel de catalizador y estimulador para que las empresas del Estado pasen a un nivel más alto de competitividad.

La misión del Estado cambia a medida que progresa la economía, para esto, deben tener en cuenta factores como estimular la demanda de productos avanzados, confrontando a los sectores que deben tener tecnologías de vanguardia, a través de proyectos cooperativos simbólicos, estableciendo premios que recompensan la calidad y la aceleración del ritmo de las innovaciones tecnológicas.

La clase política comete errores en sus decisiones al intentar manejar la estructura industria del país, proteger el mercado durante largo tiempo, rendirse a las presiones políticas para aislar a algunos actores. Se requieren amplios esfuerzos de descentralización y dinamismo, que permitan mejorar la gestión y dejar de ser una influencia negativa.

Son aspectos que retrasan la innovación tecnológica, la relación existente entre tiempo competitivo de las empresas y tiempo político de los gobernantes, son limitantes, por la "temporalidad", vista se considera que para lograr una marcada ventaja competitiva de un sector se requiere más de un decenio de tiempo que permita integrar las mejoras de las destrezas humanas, inversión en productos y procesos, creación de organización empresarial, penetración de mercados, etc. Como un decenio es bastante tiempo, muchos gobernantes prefieren establecer políticas que ofrezcan ventajas materializables en el corto plazo (períodos de gobierno), por ejemplo, las subvenciones, la protección y fusiones convenidas.

Los factores que se traducen en ventaja competitiva para el Estado, son avanzados, especializados, y ligados a sectores específicos como el de defensa. Debe entenderse que las políticas que 
ofrecen mayores beneficios, son demasiado lentas, costosas y exigen paciencia o generan cambios bruscos en el corto plazo.

Finalmente, debe reconocerse la política de comercio exterior del Estado en su papel de ayudar a dar acceso libre al mercado con otras naciones, el cual, para ser eficaz no debe ser un instrumento pasivo, debe tratar de abrir mercados donde la nación tenga una ventaja competitiva. Ante una barrera comercial con otra nación, se debe concentrar sus medidas en desmantelarla y no en regular importaciones o exportaciones.

\section{Referencias bibliográficas}

Ansoff, I. (1965), Corporate strategy: an analytic approach to business policy for growth and expansion

Chandler, A., (1962), Strategy and Structure: Chapters in the History of the American Industrial Enterprise

De Ríos, C. C. (2018). ¿ Cuál es la importancia de implementar estrategias en las organizaciones?. Revista científica anfibios, 1(1), 71-79.

Gutiérrez Díez, Luis Angel, Evolución de la tecnología militar y su impacto en España, Gasto militar e industrialización, España, 1995.

Hernández, H. G., Cardona, D. A., \& Del Rio, J. L. (2017). Direccionamiento Estratégico: Proyección de la Innovación Tecnológica y Gestión Administrativa en las Pequeñas Empresas. Información tecnológica, 28(5). doi:http://dx.doi.org/10.4067/S0718-07642017000500003

Mayo Muñiz, Luis, La defensa del futuro: innovación, tecnología e industria, Instituto español de estudios estratégicos, Capítulo V, España, 2011.

Miles, R.E. Y Ch.C. Snow (1978): Organizational Strategy, Structure And Process, Mcgraw-Hill.

Peinado, A. (2018). Propuesta de un plan estratégico logístico para una empresa de carácter social sin ánimo de lucro (Seccional Cali). Revista científica anfibios, 1(2), 60-68.

Perez Arrieu, J. C. (2017). Conocimiento, C\&T Y Poder Militar EN El Siglo XXI Las Guerras Del Futuro. Retrieved from Estudios de vigilancia y prospectiva tecnológica en el área de Defensa y Seguridad, Ciudad Autónoma de Buenos Aires: Escuela Superior Técnica. Centro de Estudios de Prospectiva Tecnológica Militar: http://www.ceptm.iue.edu.ar/tec1000-17/files/download/ TEC10002017.pdf

Porter, M. (1980): Competitive Strategy, The Free Press.

Rubio, J. (2018). Estrategias para brindar sustentabilidad a las líneas de comunicaciones marítimas a partir del crecimiento económico de Colombia 2010-2015: perspectiva rol de la ARC. Revista científica anfibios, 1(2), 53-59.

Sarmiento, L. M. D. (2018). Gestión estratégica de proveedores para fortalecer el proceso de compras en la línea de negocios de reparación y mantenimiento de embarcaciones en COTECMAR. Revista científica anfibios, 1(2), 44-52.

Vergara, F, Fernandez, J. C. R., Prins, Z. A., \& Gutierrez, G. C. (2019). Estrategias no convencionales de servicio: un desafío en el diseño organizacional. Revista científica anfibios, 2(1), 31-48. 